\title{
La representación de documentos y autoridades archivísticas: una mirada desde la perspectiva de la normalización
}

\section{Dunia LLanes Padrón}

Mariângela Spotti Lopes Fujita

\begin{abstract}
Posdoctorado por la Universidad Estadual Paulista (UNESP). Doctora en Biblioteconomía y Documentación por la Universidad de Salamanca, Profesora del Departamento de Ciencias de la Informaciónde la Universidad de La Habana, Cuba.
\end{abstract}

Doutora em Ciências da Comunicação pela Universidade de São Paulo. Professora Titular do Departamento de Ciência da Informação da Faculdade de Filosofia e Ciências da UNESP Campus de Marília. Pró-Reitora de Extensão Universitária da UNESP.

http://dx.doi.org/10.1590/1981-5344/2643

La Archivística, en la actualidad, peregrina por mudanzas importantes en su concepción como ciencia. Como consecuencia, la disciplina ha pasado de la inactividad en la normalización de sus tareas a la regulación de la mayoría de sus procesos. A finales del siglo $X X$, instituciones como el Consejo Internacional de Archivos $y$ prestigiosas asociaciones de archiveros desarrollaron normas para simplificar los métodos de trabajo y garantizar mejores resultados en los procesos de representación y recuperación de la información. Este trabajo tiene como propósito identificar, describir $y$ categorizar la estructura general e interna de las normas de descripción publicadas después de $\operatorname{ISAD}(G)$ e ISAAR(CPF) y que constituyen modelos para la representación de documentos y autoridades. La investigación que se presenta es de naturaleza descriptiva y se centra en el estudio de estos estándares. Los tiempos posmodernos están marcados por el acceso a la información desde múltiples perspectivas y el trabajo colaborativo entre instituciones de información, por ende, es imposible ejecutar y desenvolver procesos archivísticos que no estén normalizados. El uso de cánones para representar la información certifica la confiabilidad, la uniformidad, la accesibilidad, la disponibilidad y la apropiación de cualquier recurso de información así como 
la interoperabilidad entre sistemas de cualquier institución.

Palabras Clave: Representación y recuperación de información; Normas de descripción; Normalización.

\section{The representation the documents and authorities archivist: a look from the perspective of standardization.}

Nowadays, the archival science is going through a big changes in its conception as science. As a result, the discipline has gone from the inactivity in the normalization of their tasks to the regulatory action of most its processes. In the late twentieth century, institutions such as the International Council on Archives and prestigious associations of archivists began to develop standards to simplify working methods and ensure better results in the representation and information retrieval processes. This paper aims to identify, describe and categorize the general and internal structure of descriptive standards published after ISAD(G) and ISAAR(CPF) which constitute models for representing documents and authorities. This research is mainly of a descriptive nature and focuses on the study of international, national and regional standards published about the archival entities representation. Actually, you can access to the information from multiple perspectives and the collaborative work among information institutions, it is impossible to execute archival processes that are not standardized. The use of canons to represent information certifies the reliability, consistency, accessibility, availability and appropriation of any information source and interoperability between systems of any institution.

Keywords: Representation and retrieve information; Standards description; Standardization.

Recebido em 02.05.2016 Aceito em 12.04.2017 


\section{Introducción}

La Archivística, en los tiempos actuales, peregrina por mudanzas importantes en su concepción como ciencia. La disciplina está en un período de transición paradigmática, traspasando las fronteras del custodial hacia los horizontes pos-custodiales. Este nuevo paradigma emerge por el impacto directo de Revolución Electrónica en la Ciencia de la Información. Durante esta transición, la Archivística pasó de la inactividad en la normalización de sus tareas al ejercicio regulador de la mayoría de sus procesos. Uno de los resultados directos de estas nuevas concepciones fue el consentimiento unánime de los profesionales para elaborar metodologías uniformes que abordaran la problemática de la representación de documentos y autoridades en los sistemas electrónicos de información.

A finales del siglo $X X$, instituciones como el Consejo Internacional de Archivos (CIA) y prestigiosas asociaciones de archiveros del mundo entero comenzaron a desarrollar normas para simplificar los métodos de trabajo, ahorrar tiempo y garantizar mejores resultados en el proceso de descripción.

El cambio definitivo que marca la transición en las prácticas de representación de los contenidos fue, indudablemente, este proceso de normalización internacional. El consejo, fundamentado en las relaciones y convergencias que se establecen entre productor, funciones, documentos e instituciones de custodia, diseñó y elaboró normas internacionales para representar las entidades archivísticas. El CIA, con la publicación de su conjunto normativo, tenía como propósito establecer una estructura de datos uniforme de descripción y no una estructura de contenidos a nivel internacional. Por tanto, a las comunidades archivísticas nacionales les corresponde elaborar sus propias normas de descripción para regular los contenidos y adecuarlos a sus realidades archivísticas.

El movimiento normativo del organismo internacional tuvo un impacto inmediato en el desarrollo de normas nacionales y regionales de datos, contenidos, presentación y codificación para representar y recuperar documentos y productores. Gran Bretaña, EE.UU., Canadá, Portugal, Irlanda, Brasil, Uruguay y España (Castilla y León, Cataluña, Galicia y Aragón) son los países y comunidades, que hasta la fecha, han desarrollado estándares de descripción archivística.

El texto que se presenta tiene como propósito identificar y describir la estructura general e interna de las normas de descripción que se publicaron después de ISAD(G) e ISAAR(CPF) y que constituyen modelos para la representación de los documentos y de las autoridades archivísticas. A través de este estudio se exponen los aspectos conceptuales y organizativos que definen la estructura general de estas normas (fundamentos teóricos, entidades y sub-entidades de representación, elementos obligatorios). Se realiza, además, un análisis detallado de temas vinculados con características internas de las 
normativas (terminología, ubicación de elementos, reglas de contenido y fuentes de información).

Esta investigación es de naturaleza descriptiva y se centra en el estudio de los siguientes estándares:

a) Norma Internacional de Descripción Archivística (ISAD (G);

b) Norma Internacional sobre los Registros de Autoridades de Archivos Relativos a Instituciones, Personas y Familias (ISAAR (CPF);

c) Manual of Archival Description (MAD3);

d) Describing Archives: A Content Standards (DACS);

e) Rules for Archival Description (RAD2);

f) Orientações para a Descrição Arquivística (ODA);

g) Norma Brasileira de Descrição Arquivística (NOBRADE);

h) Manual de Descripción Multinivel (MDM);

i) Norma Española de Descripción Archivística (NEDAI - Proyecto);

j) Norma de Descripción Archivística de Cataluña (NODAC);

k) Norma Gallega de Descripción Archivística (NOGADA);

I) Norma Aragonesa para la Descripción de Autoridades de Archivos (ARANOR);

m) Norma Uruguaya de Descripción Archivística (NUDA).

Los tiempos actuales están determinados por el acceso a la información desde múltiples perspectivas y el trabajo colaborativo entre instituciones diversas de información, por tanto es imposible ejecutar y desenvolver procesos archivísticos que no estén normalizados. El uso de cánones para representar y acceder a la información asegura la autenticidad, la uniformidad, la accesibilidad, la disponibilidad y la apropiación de cualquier recurso de información así como la interoperabilidad entre sistemas de cualquier institución.

\section{Estructura general de las normas de descripción archivística}

Este apartado tiene como objetivo realizar una caracterización de los aspectos más representativos de la estructura general de $\operatorname{ISAD}(G)$, ISAAR(CPF), MAD3, DACS, RAD2, ODA, NEDA-I, MDM, NOGADA, NODAC, NOBRADE, NUDA y ARANOR. Para garantizar la uniformidad del trabajo y obtener resultados uniformes se creyó conveniente establecer categorías de análisis sustentado en los siguientes elementos:

a) fundamentos teóricos;

b) entidades archivísticas; 


\section{c) sub-entidades archivísticas; \\ d) elementos obligatorios}

Después de observar y estudiar cada uno de estos elementos en las normas objeto de estudio se elaboraron un conjunto de tablas y gráficos acompañados de textos explicativos que facilitan la visión del estudio. Seguidamente, se muestran de forma sistemática los resultados obtenidos.

Los primeros pasos hacia la consolidación de los principios teóricos de la descripción archivística los dio el organismo internacional. En 1992, el CIA, a través de la Comisión Ad Hoc sobre normas de descripción, da a conocer la primera versión revisada de la Declaración de Principios de Descripción Archivística. Esta declaración recoge los planteamientos teóricos por lo que se debe regir la descripción y su normalización en los tiempos contemporáneos. En esta declaración se establece cuáles son los propósitos de la descripción archivística: creación de descripciones uniformes, pertinentes y explícitas, facilitar la recuperación y el intercambio de información sobre materiales de archivos, posibilitar el intercambio de registros de autoridad y la integración de las descripciones realizadas por distintos archivos o instituciones en un sistema integrado de información (CONSEJO INTERNACIONAL DE ARCHIVOS, 1992).

Posteriormente, los archiveros de EE.UU y Canadá trabajaron en un proyecto común conocido como CUSTARD (Canada-US Task Force on Archival Description). Como consecuencia de este trabajo conjunto surgió la Declaración de Principios de este Proyecto. Las declaraciones del CIA y CUSTARD recogen los principios fundamentales de la descripción archivística y han influido, positivamente, en las bases conceptuales y teóricas de los nuevos proyectos normativos. En la siguiente tabla se ejemplifican los principios teóricos expuestos por el CIA, el proyecto CUSTARD y el resto de proyectos normativos publicados.

Tabla 1 - Principios teóricos de la descripción archivística normalizada

\begin{tabular}{|c|c|}
\hline & PRINCIPIOS TEÓRICOS DE LA DESCRIPCIÓN ARCHIVÍSTICA \\
\hline 1 & $\begin{array}{l}\text { El principio de respeto a los fondos es la base de la organización y de la descripción (Principio de procedencia y } \\
\text { orden natural) }\end{array}$ \\
\hline 2 & La descripción tiene lugar después de que se haya completado la organización. \\
\hline 3 & La descripción refleja la clasificación. \\
\hline 4 & Los niveles de organización están determinados por los niveles de descripción. \\
\hline 5 & Los productores de materiales archivísticos, tanto como los materiales en sí mismos, también deben ser descritos. \\
\hline 6 & Las descripciones se presentan de lo general a lo específico y se especifican niveles de jerarquía. \\
\hline 7 & La descripción archivística se basa en los principios de la descripción multinivel. \\
\hline 8 & La descripción se aplica a todos los materiales de archivo con independencia de su forma o soporte. \\
\hline 9 & $\begin{array}{l}\text { La descripción se aplica en todas las fases del ciclo vital de los documentos de archivo; pueden variar los elementos y } \\
\text { la exhaustividad usado en cada uno de las fases. }\end{array}$ \\
\hline 10 & $\begin{array}{l}\text { Los principios de la descripción archivística se aplican igualmente a los documentos creados por organizaciones, por } \\
\text { individuos o familias. }\end{array}$ \\
\hline 11 & Las normas de descripción pueden utilizarse para describir colecciones y documentos sueltos. \\
\hline 12 & Las normas deben facilitar la profundidad en las descripciones. \\
\hline
\end{tabular}




\begin{tabular}{|c|c|}
\hline & \\
\hline 16 & $\begin{array}{l}\text { Las descripciones de todas las entidades archivísticas deben estar integradas y deben responder a las mismas reglas de } \\
\text { descripción. }\end{array}$ \\
\hline 15 & $\begin{array}{l}\text { La unidad de descripción más amplia es la determinada por el servicio de archivo de cada país (Acervo, Grupo de } \\
\text { Fondos, Fondo). }\end{array}$ \\
\hline 16 & La descripción del fondo se representa como un todo y este se describe antes de sus partes. \\
\hline 17 & $\begin{array}{l}\text { Cada descripción, independientemente del nivel, se compone de un conjunto ordenado de elementos. La información } \\
\text { que se ofrece en cada nivel de descripción debe ser pertinente al nivel que se está describiendo, es decir, a cada nivel } \\
\text { le corresponde una información adecuada y específica. }\end{array}$ \\
\hline 18 & Las descripciones deben ser representaciones exactas y fieles de la entidad archivística que se describe. \\
\hline 19 & Las descripciones deben contar con términos indizados para facilitar la recuperación. \\
\hline 20 & $\begin{array}{l}\text { Las descripciones de los productores deben constituir registros de autoridades, y estos, a su vez, deben estar formados } \\
\text { por elementos informativos que identifiquen y describan la entidad y, también, deben remitir a otros registros de } \\
\text { autoridad relacionados. }\end{array}$ \\
\hline 2 & $\begin{array}{l}\text { Las descripciones de las autoridades archivísticas deben ser realizadas y mantenidas separadas de las descripciones de } \\
\text { los documentos de archivos. }\end{array}$ \\
\hline 2 & $\begin{array}{l}\text { Las descripciones de las autoridades archivísticas y de los documentos de archivos, aunque se realicen } \\
\text { separadamente, deben interrelacionarse y complementarse. }\end{array}$ \\
\hline 23 & $\begin{array}{l}\text { Los registros de autoridades archivísticas deben describir relaciones entre las autoridades archivísticas, y los } \\
\text { documentos y entre las autoridades archivísticas y otro tipo de recurso. }\end{array}$ \\
\hline 2 & $\begin{array}{l}\text { La descripción de las autoridades archivísticas debe sustentarse en el uso de puntos de acceso normalizados y el } \\
\text { control de autoridades. }\end{array}$ \\
\hline 2 & $\begin{array}{l}\text { La descripción de las funciones debe documentar las relaciones entre diferentes funciones, entre dichas funciones y } \\
\text { las instituciones que las llevaron a cabo, así como entre tales funciones y los documentos que originaron. }\end{array}$ \\
\hline 2 & $\begin{array}{l}\text { La descripción de las funciones debe complementar la descripción de documentos y los registros de autoridad, para } \\
\text { garantizar la formación de sistemas de descripción archivísticas. }\end{array}$ \\
\hline
\end{tabular}

Fuente: Elaboración propia.

En las normas nacionales y regionales publicadas después de $\operatorname{ISAD}(\mathrm{G})$ se toman en consideración la declaración de principios del CIA y CUSTARD. Los nuevos proyectos incluyen la mayoría de los principios expuestos en estas dos declaraciones pero realizan sus propias formulaciones teóricas, adicionando nuevos preceptos $y$, en algunos casos, obviando otros que no se corresponden con las realidades y las tendencias archivísticas de cada país o región. Los estándares no pueden ser, exclusivamente, compendios técnicos; las normativas deben ser un fiel reflejo de la consistencia teórica que ha alcanzado la Archivística en los últimos años. La representación de la información, en los entornos electrónicos, debe presentarse en sistemas donde estén definidas las interrelaciones entre las diversas entidades archivísticas. Estas relaciones son las que determinan el funcionamiento eficiente de los sistemas integrados de información.

Los usuarios actuales precisan contar con sistemas donde la información esté relacionada, vinculada y con consistencia en sus contenidos, por tanto, es preciso diseñar sistemas de representación y recuperación basados en modelos conceptuales que incluyan varias entidades archivísticas (documentos, autoridades, funciones, materias, instituciones de custodia). Las normas de descripción deben desarrollar estructuras de datos y contenidos para distintas entidades. En este estudio se identificaron las entidades archivísticas que están contenidas en los estándares publicados. 
Tabla 2 - Entidades archivísticas en normas internacionales, nacionales y regionales

\begin{tabular}{|l|c|c|c|}
\hline \multicolumn{1}{|c|}{ NORMAS } & ENTIDADES DE DESCRIPCIÓN \\
\hline ISAD(G) & Documentos & Productores & \\
\hline DACS & Documentos & Productores & Materias \\
\hline ODA & Documentos & Productores & Materias \\
\hline MDM & Documentos & & Materias \\
\hline RAD2 & Documentos & & \\
\hline MAD3 & Documentos & & \\
\hline NOBRADE & Documentos & & \\
\hline NEDAI & Documentos & & \\
\hline NODAC & Documentos & & \\
\hline NOGADA & Documentos & Productores & \\
\hline IGAD & Documentos & Productores & \\
\hline NUDA & Documentos & & \\
\hline ISAAR(CPF $)$ & & & \\
\hline ARANOR & & & \\
\hline
\end{tabular}

Fuente: Elaboración propia.

Tal y como se puede observar en la tabla anterior, la mayoría de las normas incluye estructuras favorables para la representación de documentos archivísticos y, sólo, en cinco normas se desarrollan los contenidos para la creación de registros de autoridades. Por su parte, la normalización de los puntos de acceso es, también, un tema poco abordado en la Archivística, aunque es válido aclarar que, en los últimos años, se ha publicado estándares nacionales que regulan los puntos de acceso vinculados a los nombres de instituciones, personas y familias. Los sistemas unificados de información archivística deben incluir, siempre, datos sobre los documentos y sus productores $y$, opcionalmente, de otros tipos de entidades. Los sistemas actuales tienden a ser más completos e incluir y relacionar la mayoría de las entidades.

Además de definir en una norma las entidades, también, es preciso determinar cuáles son las sub-entidades que serán incluidas en las estructuras. Los documentos de archivo, los agentes y las funciones son las únicas entidades que presentan subtipos. Un subtipo de entidad es, según el modelo conceptual de la (MINISTERIO DE EDUCACIÓN, CULTURA Y DEPORTE, 2011, p. 21): "[...] una categoría de un subconjunto de entidades pertenecientes al mismo tipo que poseen los mismos atributos y relaciones. También denominado subclase de tipo de entidad".

El CIA ha definido subtipos de entidades a nivel internacional que deben ser adoptadas por las comunidades nacionales pero, a la vez, pueden incluirse otras de acuerdo a las necesidades de cada región (véase tabla 3). 
Tabla 3 - Entidades y Sub-entidades archivísticas propuestas por el CIA para documentos y productores

\begin{tabular}{|c|c|}
\hline \hline \multicolumn{2}{|c|}{ ENTIDADES/SUBENTIDADES ARCHIVÍSTICAS } \\
(CIA)
\end{tabular}

Fuente: Elaboración propia.

Los subtipos de entidades que se muestran en la tabla anterior son los delimitados en las normas internacionales. Las normas nacionales y regionales han propuestos otros subtipos, principalmente para el caso de la entidad documento de archivo (véase tablas 4,5 y 6). La definición de sub-entidades depende, en la mayoría de los casos, de las prácticas descriptivas de cada comunidad archivística. Algunas tipos de entidades no presentan subtipos como es el caso de: entidades de custodia, normas, materias o lugares y otras. Los estándares deben presentar estructuras de datos y contenidos de acuerdo a las entidades y sub-entidades definidas en sus modelos teóricos.

Tabla 4 - Tipos de sub-entidades archivísticas en normas anglosajonas

\begin{tabular}{|c|c|c|}
\hline \multicolumn{3}{|c|}{$\begin{array}{c}\text { SUB-ETIDADES ARCHIVÍSTICAS PROPUESTAS EN NORMAS } \\
\text { ANGLOSAJONAS }\end{array}$} \\
\hline$M A D 3$ & $D A C S$ & $R A D 2$ \\
\hline \multicolumn{3}{|c|}{ DOCUMENTOS } \\
\hline Acervo & Grupo de fondos & Fondos \\
\hline Grupo de fondos & Fondos & Series \\
\hline Fondos & Series & Documento Compuesto \\
\hline Sub-fondos & Documento Compuesto & Documento Simple \\
\hline Series & Documento Simple & Colecciones \\
\hline Documento Compuesto & Colecciones & \\
\hline \multicolumn{3}{|l|}{ Documento Simple } \\
\hline \multicolumn{3}{|c|}{ PRODUCTOR } \\
\hline \multirow{3}{*}{$\begin{array}{l}\text { No contiene sub-entidades para el } \\
\text { productor }\end{array}$} & Instituciones & \multirow{3}{*}{$\begin{array}{l}\text { No contiene sub-entidades para el } \\
\text { productor }\end{array}$} \\
\hline & Personas & \\
\hline & Familias & \\
\hline
\end{tabular}

Fuente: Elaboración propia. 
Tabla 5 - Tipos de sub-entidades archivísticas en normas de Hispanoamérica

\begin{tabular}{|c|c|c|c|c|c|}
\hline \multicolumn{6}{|c|}{$\begin{array}{c}\text { SUB-ETIDADES ARCHIVÍSTICAS PROPUESTAS EN NORMAS } \\
\text { HISPANOAMERICANAS }\end{array}$} \\
\hline NEDA & 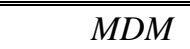 & NODAC & "NOGADA & ARANOR & NUDA \\
\hline \multicolumn{6}{|c|}{ DOCUMENTOS } \\
\hline Grupo de Fundos & Fondos & Fondos & Fondos & \multirow{12}{*}{$\begin{array}{l}\text { No contiene } \\
\text { estructura de datos } \\
\text { para documento }\end{array}$} & Fondos \\
\hline Fondos & Sub-fundo & Sub-fondo & Sección & & Sub-fondo \\
\hline $\begin{array}{l}\text { División de } \\
\text { Fondos }\end{array}$ & Sección & Series & Serie & & Series \\
\hline Serie & Sub-sección & Grupo de Series & Sub-serie & & $\begin{array}{l}\text { Documento } \\
\text { Compuesto }\end{array}$ \\
\hline Sub-serie & Serie & $\begin{array}{l}\text { Unidad de } \\
\text { localización }\end{array}$ & Fracción de Serie & & $\begin{array}{l}\text { Documento } \\
\text { Simple }\end{array}$ \\
\hline Serie facticia & Sub-serie & $\begin{array}{l}\text { Documento } \\
\text { Compuesto }\end{array}$ & $\begin{array}{l}\text { Documento } \\
\text { Compuesto }\end{array}$ & & Colecciones \\
\hline $\begin{array}{l}\text { Fracción de } \\
\text { Serie/Fracción de } \\
\text { Sub-serie }\end{array}$ & $\begin{array}{l}\text { Unidad de } \\
\text { localización }\end{array}$ & $\begin{array}{l}\text { Documento } \\
\text { Simple }\end{array}$ & $\begin{array}{l}\text { Documento } \\
\text { Simple }\end{array}$ & & \\
\hline $\begin{array}{l}\text { Documento } \\
\text { Compuesto }\end{array}$ & $\begin{array}{l}\text { Documento } \\
\text { Compuesto }\end{array}$ & & & & \\
\hline $\begin{array}{l}\text { Documento } \\
\text { Simple }\end{array}$ & $\begin{array}{l}\text { Documento } \\
\text { Simple }\end{array}$ & & & & \\
\hline Colecciones & & & & & \\
\hline $\begin{array}{l}\text { División de } \\
\text { Colección }\end{array}$ & & & & & \\
\hline $\begin{array}{l}\text { Componente } \\
\text { Documental } \\
\end{array}$ & & & & & \\
\hline \multicolumn{6}{|c|}{ PRODUCTOR } \\
\hline \multirow{3}{*}{$\begin{array}{l}\text { No contiene sub- } \\
\text { entidades para } \\
\text { productor }\end{array}$} & Instituciones & \multirow{3}{*}{$\begin{array}{l}\text { No contiene } \\
\text { sub-entidades } \\
\text { para productor }\end{array}$} & \multirow{3}{*}{$\begin{array}{l}\text { No contiene sub- } \\
\text { entidades para } \\
\text { productor }\end{array}$} & Instituciones & \multirow{3}{*}{$\begin{array}{l}\text { No contiene } \\
\text { sub-entidades } \\
\text { para productor }\end{array}$} \\
\hline & Personas & & & Personas & \\
\hline & Familias & & & Familias & \\
\hline
\end{tabular}

Fuente: Elaboración propia.

Tabla 6 -Tipos de sub-entidades archivísticas en normas de Portugal y Brasil

\begin{tabular}{|c|c|}
\hline \multicolumn{2}{|c|}{$\begin{array}{c}\text { SUB-ETIDADES ARCHIVISTICAS PROPUESTAS EN NORMAS } \\
\text { LUSO-BRASILENAA }\end{array}$} \\
\hline$O D A$ & NOBRADE \\
\hline \multicolumn{2}{|c|}{ DOCUMENTOS } \\
\hline Grupo de Fondos & Acervo \\
\hline Fondos & Acervo de la sub-unidad de custodia \\
\hline Sub-fondos & Fondos/Colección \\
\hline Sección & Sección \\
\hline Sub-sección & Sub-sección \\
\hline Serie & Serie \\
\hline Sub-serie & Sub-serie \\
\hline Unidad de Instalación & Dossier/Proceso \\
\hline Doc. Compuesto & Doc. Simple \\
\hline \multicolumn{2}{|l|}{ Doc. Simple } \\
\hline \multicolumn{2}{|l|}{ Colección } \\
\hline \multicolumn{2}{|c|}{ PRODUCTOR } \\
\hline Instituciones & \multirow{3}{*}{ No contiene sub-entidades para productor } \\
\hline Personas & \\
\hline Familias & \\
\hline
\end{tabular}

Fuente: Elaboración propia. 
En la estructura general de estas normativas, un tema central es la designación de los elementos obligatorios que serán utilizados para representar cada una de las entidades archivísticas. Los elementos obligatorios facilitan el intercambio de información entre diferentes instituciones tanto a nivel nacional como internacional; además, brindan la información necesaria para describir el contenido de las unidades de descripción a un nivel básico. $\operatorname{En} \operatorname{ISAD}(G)$ se plantea que: "se pueden utilizar todos los elementos de descripción establecidos en una norma, pero sólo una parte de ellos debe, obligatoriamente, utilizarse en todos los casos". (ISAD-G, 2000, p. 15).

En las normas internacionales se delimitan, manifiestamente, los elementos mínimos obligatorios a utilizar para la descripción de documentos y autoridades (véase tabla 5).

Tabla 7 - Elementos obligatorios para documentos y productores propuestos por el CIA

\begin{tabular}{|l|l|}
\hline \multicolumn{2}{|c|}{$\begin{array}{c}\text { ELEMENTOS OBLIGATORIOS } \\
\text { (CIA) }\end{array}$} \\
\hline \multicolumn{1}{|c|}{ DOCUMENTOS } & \multicolumn{1}{c|}{ PRODUCTORES } \\
\hline Código de Referencia & Tipo de entidad \\
\hline Título & Forma Autorizada del Nombre \\
\hline Fechas & Fechas de Existencia \\
\hline Nivel de Descripción & Identificador del Registro de autoridad \\
\hline Volumen y Soporte & \\
\hline Productor & \\
\hline
\end{tabular}

Fuente: Elaboración propia.

Las normas nacionales y regionales incluyen los elementos obligatorios que han considerado pertinentes siempre conservando los elementos de la propuesta internacional pues esto garantiza la interoperabilidad entre los sistemas de descripción. En las tablas siguientes $(6,7$ y 8$)$ se ejemplifican los elementos considerados por las diferentes comunidades archivísticas. La mayoría incluye los elementos propuestos en ISAD(G) e ISAAR(CPF) pero, también, se aprecian algunas diferencias marcadas por la adición de otros elementos.

Tabla 8 - Elementos obligatorios para la entidad documento en normas anglosajonas

\begin{tabular}{|l|l|l|}
\hline \multicolumn{2}{|c|}{ ELEMENTOS OBLIGATORIOS PARA LA ENTIDAD DOCUMENTO } \\
NORMAS ANGLOSAJONAS \\
\hline \multicolumn{1}{|c|}{ MAD3 } & \multicolumn{1}{|c|}{ DACS } & \\
\hline Código de Referencia & Código de Referencia & \multirow{2}{*}{ RAD2 } \\
\hline Título & Título & \multirow{2}{*}{ No proponen elementos obligatorios } \\
\hline Fechas & Fechas & \\
\hline Nivel de Descripción & Nivel de Descripción & \\
\hline $\begin{array}{l}\text { Extensión de la unidad de } \\
\text { descripción }\end{array}$ & Extensión de la unidad de descripción \\
\hline & Nombre del productor & \\
\hline
\end{tabular}




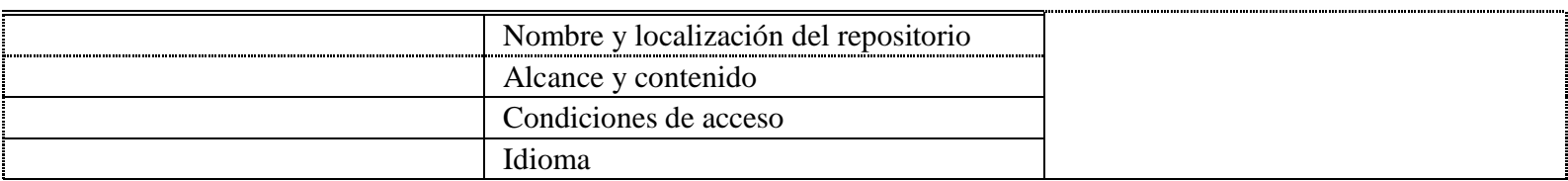

Fuente: Elaboración propia.

Tabla 9- Elementos obligatorios para documentos y productores en normas de Hispanoamérica

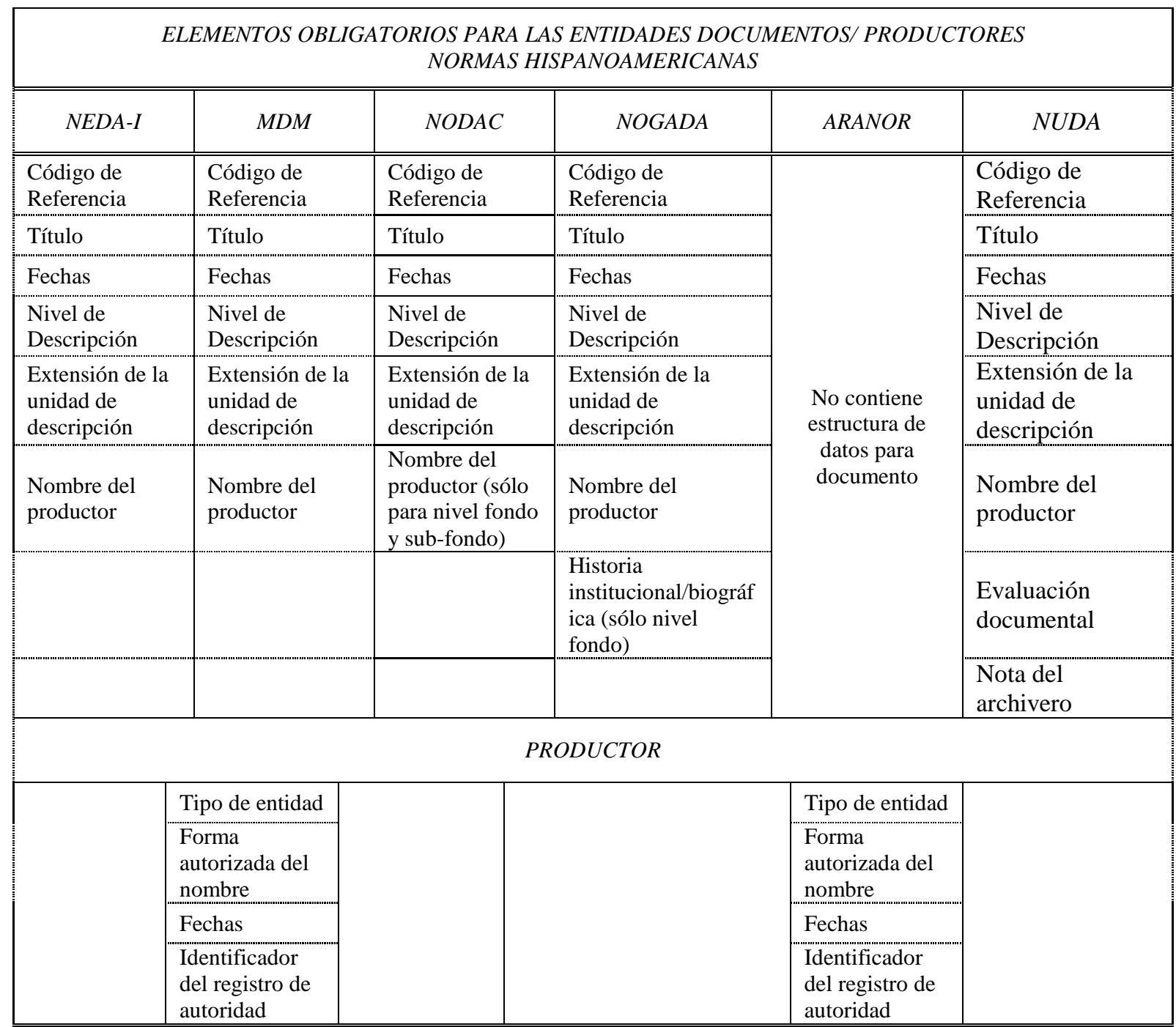

Fuente: Elaboración propia. 
Tabla 10- Elementos obligatorios para documentos y productores en normas de Portugal y Brasil

\begin{tabular}{|l|l|}
\hline \multicolumn{2}{|c|}{$\begin{array}{c}\text { ELEMENTOS OBLIGATORIOS PARA LAS ENTIDADES DOCUMENTOS / PRODUCTORES } \\
\text { NORMAS LUSO-BRASILENES }\end{array}$} \\
\hline \multicolumn{1}{|c|}{ ODA } & \multicolumn{1}{|c|}{ NOBRADE } \\
\hline \hline Código de Referencia & Código de Referencia \\
\hline Título & Título \\
\hline Fechas & Fechas \\
\hline Nivel de Descripción & Nivel de Descripción \\
\hline Extensión de la unidad de descripción & Extensión de la unidad de descripción \\
\hline Nombre del productor (sólo para nivele fondo) & $\begin{array}{l}\text { Condiciones de acceso (sólo para nivel acervo, fondo y } \\
\text { colección) }\end{array}$ \\
\hline & PRODUCTOR \\
\hline Tipo de entidad & \\
\hline Forma autorizada del nombre & \\
\hline Fechas \\
Identificador del registro de autoridad
\end{tabular}

Fuente: Elaboración propia.

El conocimiento de algunos de los aspectos generales que caracterizan la estructura de un estándar es determinante para realizar representaciones adecuadas de documentos y autoridades. Tal y como se puede observar, la mayoría de las normas nacionales y regionales logran integrar sus preceptos teóricos, entidades/sub-entidades archivísticas y los elementos obligatorios con las normas internacionales y entre ellas. La normalización del proceso de representación garantiza la unificación entre sistemas complejos, diferentes y convergentes $y$, además, garantiza el acceso a la información.

\section{Estructura interna de las normas de descripción archivística}

Este acápite tiene como propósito exponer y explicar la presentación y organización interna de los estándares. Para garantizar la consonancia del estudio, el análisis se fundamenta en los siguientes elementos:

a) elementos de información;

b) ubicación de elementos;

c) regulación de contenidos;

d) fuentes de información.

Los elementos de información circunscritos en las normas publicadas son la base de este análisis. Los elementos forman las estructuras de datos que representan las entidades archivísticas. Según ISAD(G), "la descripción archivística, en el sentido más amplio del término, se refiere a cada uno de sus elementos de información con independencia de la fase de gestión en el que se identifique o se establezca" (ISAD-G, 2000, p. 9). 
Por tanto, la definición de elementos es primordial en la realización de la descripción.

$\operatorname{ISAD}(\mathrm{G})$ delimitó veintiséis elementos de información que pueden combinarse entre sí para realizar la descripción de los documentos (ISADG, 2000, p. 10). Esta estructura de datos ha sido adoptada, en su generalidad, por las nomas nacionales y regionales. Después de un análisis minucioso de los estándares, se puede exponer que para la representación de los documentos, se identificaron 58 elementos, de ellos 26 se corresponden con los de ISAD(G). RAD2, MAD3, DACS y NOBRADE incluyen, además, nuevos elementos que se corresponden con sus realidades archivísticas.

Las reglas de Canadá, por ejemplo, proponen un conglomerado de elementos provenientes de las descripciones bibliográficas. Los archiveros canadienses en su afán de proponer reglas para diversos tipos de documentos adoptaron las áreas y los elementos de información de las AACR2 aunque se incluyen, también, los elementos de la descripción archivística. MAD3, por su parte, además de los elementos de $\operatorname{ISAD}(\mathrm{G})$, presenta un sector que recoge información relacionada con la gestión y la administración de los documentos. Los datos son de uso interno y facilitan las actividades de gestión administrativa en los archivos.

DACS, sólo, incluye un elemento adicional pero importante para los nuevos contextos digitales, se trata del elemento nombre y ubicación del repositorio. En la actualidad, la gestión y los servicios de archivos deben adaptarse a una sociedad marcada por la presencia de los documentos electrónicos. Por consiguiente, los repositorios digitales son un tema determinante en la representación, preservación y gestión de entidades archivísticas. La norma brasileña, también, contempla algunas modificaciones; adiciona un elemento relacionado con puntos de acceso e indización. Este aspecto no fue abordado en las normas internacionales, por tanto la comisión de normalización brasileña entendió conveniente regular el uso de los puntos de acceso desde la estructura de datos de la norma.

En relación a la representación de los productores archivísticos, en ISAAR(CPF), se conciertan 31 elementos de información para elaborar registros de autoridades vinculados a productores (institución, familia o persona). Estos elementos fueron aceptados por la comunidad internacional, pues en su conjunto representan de forma sistematizada las autoridades archivísticas.

La normalización de la representación de productores ha tenido un desarrollo más lento si se compara con la regulación de documentos. La confección de normas para la creación de registros de autoridades no ha sido un proceso tan vertiginoso. La descripción archivística tiene como finalidad crear sistemas de información donde interactúen y se interrelacionen informaciones de entidades e instituciones diferentes. Por tanto, crear estándares que regulen los datos y los contenidos de autoridades archivísticas es una necesidad y una tarea pendiente en varios países. EE.UU, Portugal y España, a partir de la experiencia 
internacional, publicaron reglas para definir los datos y los contenidos de los registros de autoridades. Resulta de interés, entonces, conocer la propuesta de estos países en relación a los elementos de información.

En DACS, ODA, MDM y ARANOR se identificaron un total de 39 elementos para completar registros de autoridades, de ellos 31 se corresponden con los de ISAAR(CPF). Las cuatros normas publicadas incluyeron en su mayoría los elementos internacionales.

El manual de descripción castellano - leonés presenta las diferencias más notables, precisamente por su condición de manual y no de estándar. Los autores del manual no incluyeron área de relaciones por lo que estos elementos están ausentes en la estructura, sólo, contiene un elemento relacionado con este tema: puntos de acceso de autoridad relacionados. El MDM desarrolla los elementos obligatorios de ISAAR(CPF) y otros de importancia para crear un registro de autoridad. No obstante, el manual no sigue, exactamente, la estructura de datos internacional, omite elementos y adiciona otros que son novedosos para la representación de productores.

DACS incluye, como en la descripción de documentos, un elemento vinculado a los repositorios. Por su parte, la norma aragonesa no contiene, aún, un capítulo que incluya las relaciones de registros de autoridad con otros recursos de información.

En relación a la ubicación de los elementos, se observa que las normas internacionales estructuran la información en áreas y varias normas nacionales han seguido este ejemplo. ODA, NOBRADE, NEDA-I, MDM, NODAC, NOGADA, NUDA y ARANOR organizan la información como en las propuestas internacionales, es decir, en áreas de información. DACS, RAD2 y MAD3 estructuran la información en capítulos e incluyen la mayoría de las áreas propuestas en $\operatorname{ISAD}(\mathrm{G})$ e ISAAR(CPF).

El consejo no exige seguir la misma estructura organizativa en las normas nacionales, es por ello que otros proyectos presentan otras formas de organización e incluso con áreas diferentes a las propuestas internacionales. En la figura 1 y la figura 2 se ilustran las áreas de información usadas por los estándares publicados para organizar la información referente a documentos y autoridades.

Sobre la ubicación de la información destinada a la representación de documentos, se puede decir que las áreas de contexto y contenido/estructura de $\operatorname{ISAD}(\mathrm{G})$ son las que presentan un mayor número de opciones diferentes para situar la información. En algunos estándares se definieron nuevas áreas para ubicar información contextual o de contenido pues se consideró que estos datos tenían carácter administrativo/práctico y las áreas de la normativa internacional no respondían a este fin. En otros casos, la creación de nuevas áreas se debe, principalmente, a los modelos de estructura bibliográfica que siguen determinadas normas nacionales. La inclusión de nuevas áreas se debe, principalmente, a que las comisiones de trabajo decidieron seguir los modelos de estructuras de normas anteriores de descripción (APPM, RAD, MAD O AACR2). 
Las normas anglosajonas son las que presentan las mayores divergencias en la estructura organizativa para los documentos. Es válido recordar que en Gran Bretaña, EE.UU y Canadá ya existían normas de descripción antes del surgimiento de $\operatorname{ISAD}(G)$ y estas fueron referencias para la creación de las nuevas ediciones. Un factor determinante que marca estas diferencias es, asimismo, la influencia de las normas de catalogación bibliográfica en los estándares anglosajones.

El centro de análisis, para conocer la ubicación de los elementos en el caso de la descripción de productores, lo constituyen las áreas de descripción de ISAAR(CPF). En la figura 2 se observan las áreas utilizadas para organizar la información referente a los productores. La norma internacional propone cinco áreas que han sido respetadas en, su mayoría, por los proyectos publicados. La generalidad de las áreas nuevas localizadas (formas del nombre, control de autoridad, descripción de instituciones, familias y personas y administración del registro de autoridad) sólo presentan cambios en la denominación porque mantienen los mismos objetivos y elementos de ISAAR(CPF). En las áreas de descripción y control se presentan dos áreas respetivamente diferentes, se trata de las áreas de información y de notas; estas dos áreas provienen del manual de Castilla y León que presenta una organización diferente a la propuesta en el estándar internacional.

Las diferencias más notables sobre estructura organizativa en estándares de descripción de los productores están presentes en DACS y MDM. Es válido resaltar que en la propuesta estadounidense sólo varía la forma de las denominaciones porque incluye la mayoría de los datos de ISAAR(CPF). El manual castellano, en cambio, las diferencias son más notables pues sigue una estructura organizativa distinta.

La regulación de los contenidos en la descripción de documentos y autoridades es uno de los temas más importantes dentro de la regulación de este proceso. Las reglas generales y específicas son las que determinan el cómo y el por qué de la representación de la información. La solidez de una norma, o su nivel de eficiencia/calidad, debe evaluarse a través de índices de normalización e innovación aplicados a sus contenidos. Los estándares descriptivos necesitan compilar un número suficiente de reglas que faciliten la resolución de todo tipo de problemas durante el proceso de representación de la información en las instituciones archivísticas. En este trabajo se considera conveniente exponer cuáles son las normas nacionales y regionales publicadas, hasta la fecha, que presentan mayores índices de normalización, innovación o coincidencia en relación a la regulación de sus contenidos. Para realizar el análisis de contenidos, se utilizan como referencia los indicadores definidos y presentados en la Tesis Doctoral de Llanes Padrón (2011), que evalúa los índices de normalización, coincidencia e innovación en la regulación de los contenidos de las normas publicadas para describir documentos. Estos mismos indicadores serán usados, también, para examinar estos aspectos en las normas creadas para confeccionar registros de autoridades. 
Figura 1 - Áreas de información para la entidad documento en normas internacionales, nacionales y regionales

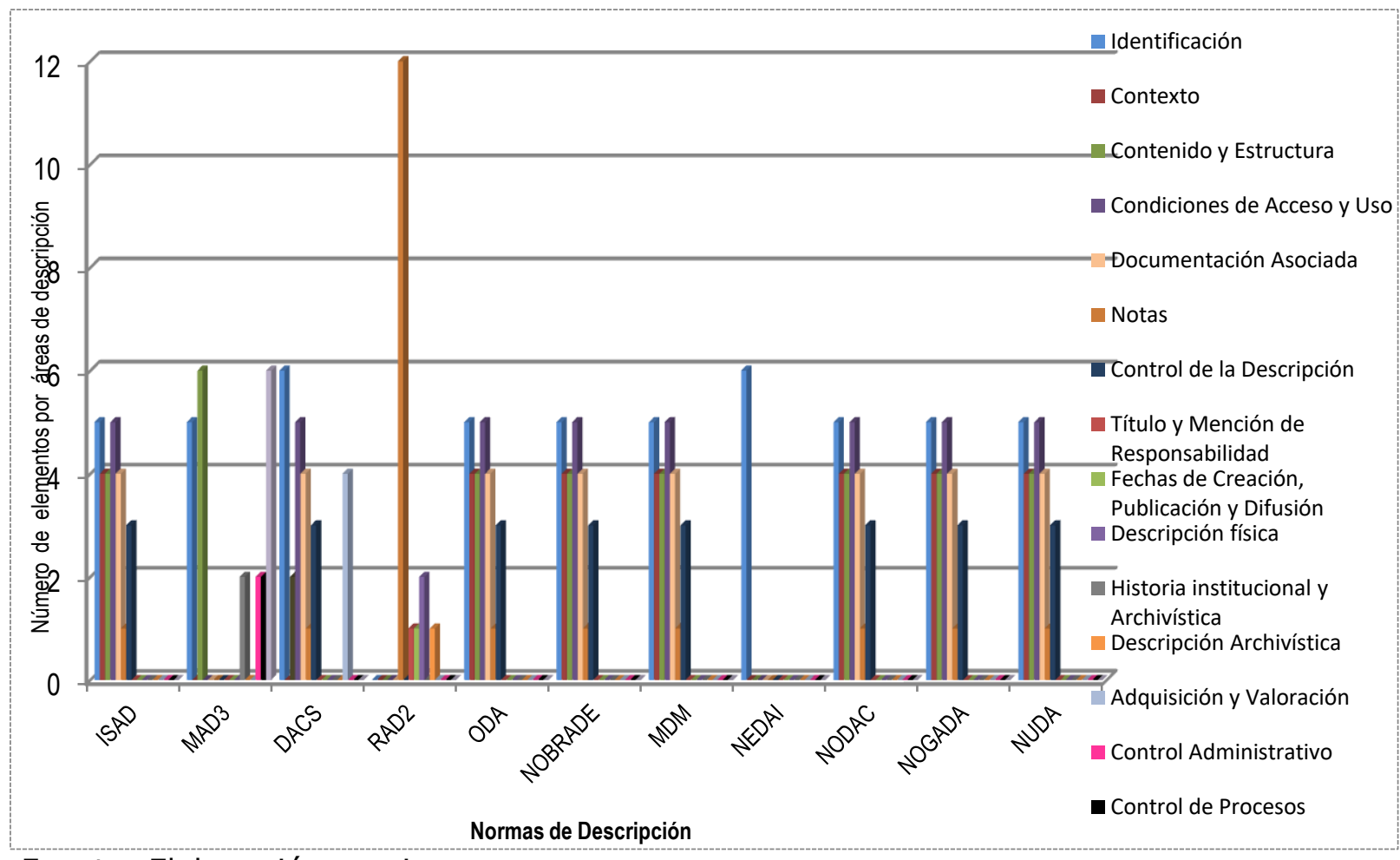

Fuente: Elaboración propia.

Figura 2 - Áreas de información para la entidad productor en normas internacionales, nacionales y regionales

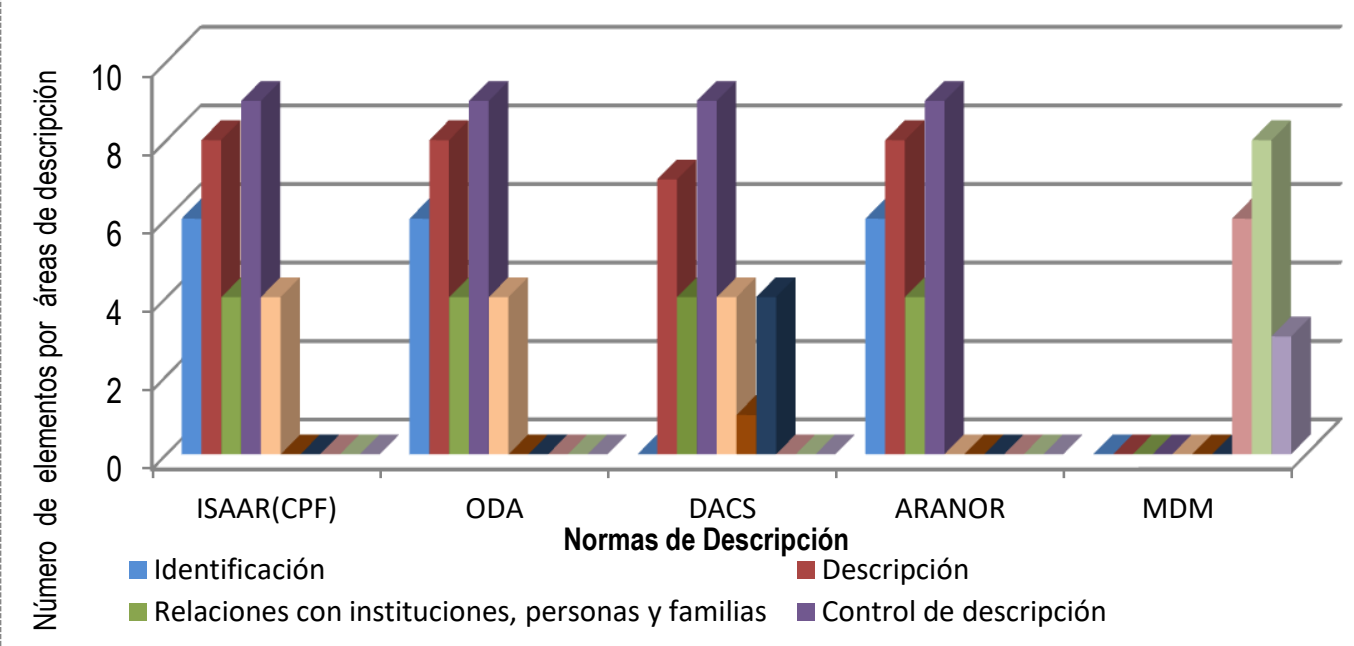

Fuente: Elaboración propia. 
A continuación se citan los índices utilizados para estudiar la regulación de los contenidos en los estándares:

Índice de normalización (IN):

Objetivo: conocer las normas que regulan un mayor número de contenidos

Índice de coincidencia (IC)

Objetivo: conocer el grado de coincidencia de una norma con $\operatorname{ISAD}(\mathrm{G})$ o ISAAR(CPF)

Índice de innovación (II)

Objetivo: Conocer el grado de diferencia de una norma con $\operatorname{ISAD}(\mathrm{G})$ o ISAAR(CPF). (LLANES PADRÓN, 2011, p. 18-20) ${ }^{1}$.

A partir de estos indicadores y de su aplicación se realizó un estudio minucioso y cuantitativo de las reglas de contenidos planteadas en los estándares. Con estos valores se calcularon los promedios generales de estos índices y su comportamiento en las normas. Finalizado este trabajo de análisis, se procesaron todos los datos obtenidos $\mathrm{y}$, con ello, se procedió a elaborar algunos instrumentos gráficos que ayudan a visualizar los resultados del estudio de los contenidos.

En el gráfico de la figura 3 se pueden observar los índices de normalización, coincidencia e innovación que obtuvieron MAD3, DACS, RAD2, ODA, NOBRADE, NEDA-I, MDM, NODAC, NOGADA y NUDA en relación a la regulación de contenidos para documentos:

a) ODA, NODAC, NOGADA y NEDA-I (área de identificación y productor) son los textos que obtienen los mayores índices de normalización o lo que es lo mismos son los textos que más cuestiones proponen para regular el contenido de los elementos de información para describir la entidad documento. DACS, NOBRADE, MDM y NUDA alcanzaron valores medios de normalización en la regulación de sus contenido mientras MAD3 y RAD2 ostentan los menores valores en los porcentajes de normalización de contenidos;

b) DACS, ODA, NOBRADE, NEDA-I, MDM, NODAC, NOGADA y NUDA exhiben porcentajes de coincidencia por encima del $80 \%$ con la norma internacional, lo que significa que la mayoría de estos proyectos adoptaron las reglas de contenidos propuestos por $\operatorname{ISAD}(G)$ para la descripción de la entidad documento. MAD3 y RAD2 ostentan los menores valores en los porcentajes de coincidencia con la norma internacional;

\footnotetext{
1 Para conocer la definición, las unidades de medida y las formas de cálculo de estos índices se recomienda consultar la tesis doctoral de: LLANES PADRÓN, D. La representación normalizada de los documentos: estudio comparado de normas de descripción archivística. 2011. 766f. Tesis Doctoral (Doctorado en Biblioteconomía y Documentación) - Universidad de Salamanca, Salamanca, 2011. Disponible en: <http://gredos.usal.es/jspui/handle/10366/110754>. Acceso en: 2 nov. 2015.
} 
c) ODA, NEDAI, NODAC y NOGADA son los estándares que proponen mayor cantidad de cuestiones no coincidentes con ISAD(G) para normalizar el contenido de la descripción de documentos, es decir, son los textos más innovadores pues proponen reglas nuevas no definidas en el estándar internacional. Por su parte, NOBRADE, MDM y NUDA presentan valores medios de innovación en la normalización del contenido de sus elementos mientras MAD3, DACS y RAD2 ostentan los valores más bajos de innovación en el conjunto de normas analizadas.

En el gráfico de la figura 4 se pueden observar los índices de normalización, coincidencia e innovación que obtuvieron DACS, ODA, ARANOR y MDM en relación a la regulación de los contenidos para autoridades. A partir de esta información gráfica se alcanzaron las siguientes conclusiones:

a) ARANOR y ODA son los estándares que obtienen los mayores índices de normalización, o lo que es lo mismo, son los textos que más cuestiones proponen para regular el contenido de los elementos utilizados para representar la entidad productor, destacando la norma aragonesa con un $80 \%$ de índice normativo. DACS y el MDM ostentan menores valores en los porcentajes de normalización para la creación de registros de autoridades;

b) Las cuatro normas publicadas para la descripción de los productores presentan elevados porcentajes de coincidencia con respecto a ISAAR(CPF); lo que significa que la mayoría de estos proyectos adoptaron las reglas de contenidos formulados por la norma internacional para la creación de los registros de autoridades en los contextos archivísticos;

c) ARANOR es la norma que expone la mayor cantidad de cuestiones no coincidentes con ISAAR(CPF) para regularizar los contenidos de la descripción de productores. La norma de Aragón es la más innovadora en este sentido, pues propone reglas nuevas no referidas en el estándar internacional. Por su parte, ODA exhibe valores medios de innovación en la normalización del contenido de sus elementos mientras DACS y el MDM ostentan los valores más bajos de innovación con respecto a las nuevas reglas propuestas para la representación de productores de documentos archivísticos. 
Figura 3 - Tendencias de índices normativos en normas nacionales y regionales para la descripción de documentos

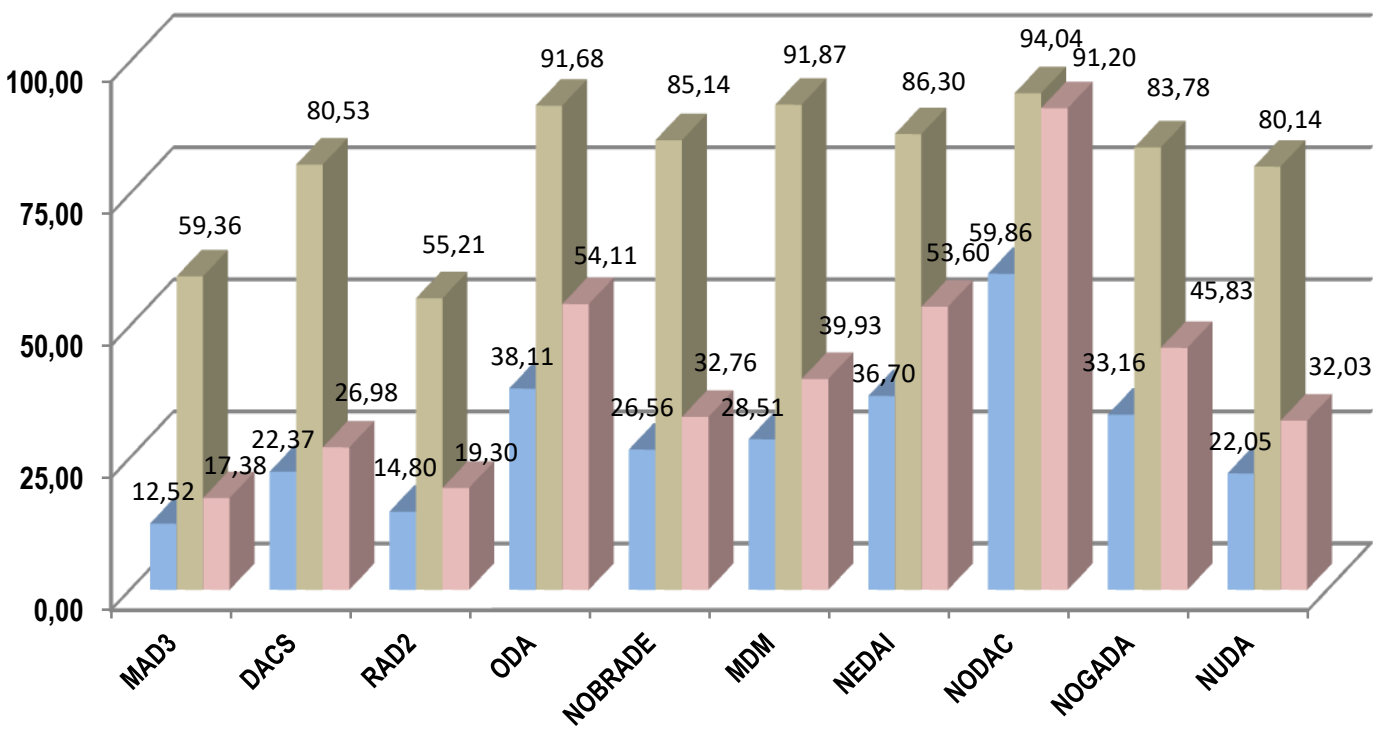

Normas de Descripción

—Índice de Normalización $\quad$ Índice de Coincidencia $\square$ Índice de Innovación

Fuente: Elaboración propia.

Figura 4 - Tendencias de índices normativos en normas nacionales y regionales para la descripción de productores

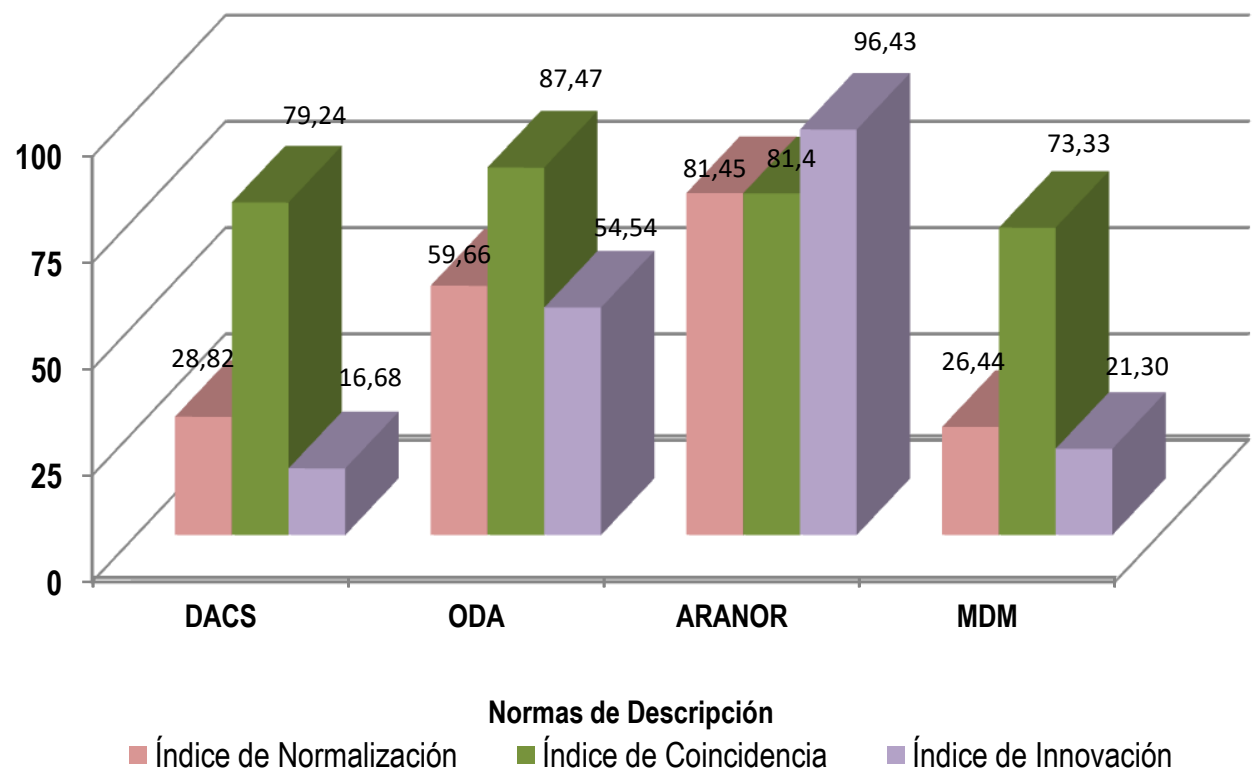

Fuente: Elaboración propia.

Estos gráficos tienen como objetivo mostrar los valores de normalización, coincidencia e innovación que obtuvieron las normas. Es 
válido destacar que este pequeño estudio cuantitativo presenta, únicamente, un carácter de orientación y referencia para la realización de este proceso.

\section{Consideraciones finales}

La Archivística del paradigma pos-custodial se caracteriza por la presencia incondicional de herramientas y software que ayudan a lidiar con el gran cúmulo de información electrónica que se produce en nuestros días. Las normativas de representación y recuperación son pertrechos que no deben faltar en el equipaje de nuestros profesionales; su conocimiento y uso es incuestionable para la realización de los procesos archivísticos, en especial la representación. Las instituciones archivísticas nacionales tienen que asumir verdaderos compromisos para desenvolver las infraestructuras necesarias para la creación y diseminación de normas de descripción.

Los nuevos profesionales de la Ciencia de la Información deben certificar la representación, la disponibilidad, la preservación, la autenticidad, la accesibilidad y la usabilidad del preciado recurso denominado "información". Para ello, sin lugar a dudas, los cánones son algunos de los vehículos más modernos que conducirán, a profesionales y usuarios, de forma rápida, eficiente y segura por las confluencias de las nuevas autopistas informacionales.

\section{Referencias}

BONAL, J.; GeNERELO, J.; TRAVESÍ DE DIEGO, C. Manual de Descripción Multinivel: propuesta de adaptación de las normas internacionales de descripción archivística. Valladolid: Junta de Castilla y León; Consejería de Educación y Cultura, 2000.

BUREAU CANADIEN DES ARCHIVISTES. Rules for archival description. Ottawa: Bureau Canadien des Archivistes, 2008. Disponible en: <http://www.cdncouncilarchives.ca/archdesrules.html>. Acceso en: 2 nov. 2015.

CONSEJO INTERNACIONAL DE ARCHIVOS. Declaración de principios sobre la descripción archivística: adoptada por la Comisión ad hoc sobre normas de descripción. Ottawa: Secretaria de la Comisión sobre Normas de Descripción, 1992.

CONSEJO INTERNACIONAL DE ARCHIVOS. ISAD (G): norma internacional para la descripción archivística. Madrid: Dirección General del Libro, Archivos y Bibliotecas, Subdirección General de los Archivos Estatales, 2000.

CONSEJO INTERNACIONAL DE ARCHIVOS. ISAAR (CPF): norma internacional sobre los registros de autoridad de archivos relativos a instituciones, personas y familias. 2. ed. Madrid: Dirección General del Libro, Archivos y Bibliotecas, Subdirección General de los Archivos Estatales, 2004. 
CONSELHO NACIONAL DE ARQUIVOS. NOBRADE: norma brasileira de descrição arquivística. Rio de Janeiro: Conselho Nacional de Arquivos, 2006.

COOK, M.; PROCTER, M. Manual of archival description. 3. ed. Aldershot: Gower, 2000.

DIRECCIÓN DEL ARCHIVO GENERAL DE LA NACIÓN (Uruguay). NUDA: norma uruguaya de descripción archivística. Montevideo: Archivo General de la Nación, 2014.

DIRECÇÃO GERAL DE ARQUIVOS (Portugal). Orientações para a descrição arquivística. 2. ed. Lisboa: Direcção Geral de Arquivos; Grupo de Trabalho de Normalização da Descrição em Arquivo, 2007.

MINISTERIO DE EDUCACIÓN, CULTURA Y DEPORTE. COMISIÓN DE NORMAS ESPAÑOLAS DE DESCRIPCIÓN ARCHIVÍSTICA (España). Modelo conceptual de descripción archivística y requisitos de datos básicos de las descripciones de documentos de archivo, agentes y funciones. Sevilla: Comisión de Normas Españolas de Descripción Archivística, 2012. Disponible en: <http://www.mcu.es/archivos/docs/NEDA_MCDA_P1_P2_20120618.pdf>. Acceso en: 2 nov. 2015.

GRUPO DE TRABAJO DE AUTORIDADES DE ARAGÓN (España). ARANOR: norma aragonesa para la descripción de autoridades de archivos. 2. ed. Aragón: Gobierno de Aragón; Departamento de Educación; Cultura y Deporte, 2014.

LLANES PADRÓN, D. La representación normalizada de los documentos: estudio comparado de normas de descripción archivística. 2011. 766f. Tesis Doctoral (Doctorado en Biblioteconomía y Documentación) Universidad de Salamanca, Salamanca, 2011. Disponible en: <http://gredos.usal.es/jspui/handle/10366/110754>. Acceso en: 2 nov. 2015.

MINISTERIO DE COMUNICACIÓN (España). NODAC: norma de descripción archivística de Cataluña. Barcelona: Departamento de Cultura, 2007.

MINISTERIO DE CULTURA (España). NOGADA: norma gallega de descripción archivística. Galicia: Junta de Galicia, Subdirección General del Patrimonio Cultural, 2008.

SOCIETY OF AMERICAN ARCHIVISTS. Statement of principles for the CUSTARD. Chicago: Society of American Archivists, 2001. Disponible en: <http://www.archivists.org/news/custardproject.asp>. Acceso en: 02 nov. 2015.

SOCIETY OF AMERICAN ARCHIVISTS. Describing archives: a content standard. 2. ed. Chicago: Society of American Archivists, 2013. 